\title{
Behavioral Deficits in Juveniles Mediated by Maternal Stress Hormones in Mice
}

\author{
Jamie Maguire ${ }^{1}$ and Istvan Mody ${ }^{2}$ \\ ${ }^{1}$ Department of Neuroscience, Tufts University School of Medicine, 136 Harrison Avenue, Boston, MA 02111, USA \\ ${ }^{2}$ Departments of Neurology and Physiology, The David Geffen School of Medicine, University of California, \\ 635 Charles Young Dr. South, Los Angeles, CA 90095, USA
}

Correspondence should be addressed to Jamie Maguire; jamie.maguire@tufts.edu

Received 18 June 2015; Revised 14 August 2015; Accepted 25 August 2015

Academic Editor: Laura Musazzi

Copyright (C) 2016 J. Maguire and I. Mody. This is an open access article distributed under the Creative Commons Attribution License, which permits unrestricted use, distribution, and reproduction in any medium, provided the original work is properly cited.

\begin{abstract}
Maternal depression has been shown to negatively impact offspring development. Investigation into the impact of maternal depression and offspring behavior has relied on correlative studies in humans. Further investigation into the underlying mechanisms has been hindered by the lack of useful animal models. We previously characterized a mouse model which exhibits depression-like behaviors restricted to the postpartum period and abnormal/fragmented maternal care (Gabrd ${ }^{-1-}$ mice). Here we utilized this unique mouse model to investigate the mechanism(s) through which maternal depression-like behaviors adversely impact offspring development. Cross-fostering experiments reveal increased anxiety-like and depression-like behaviors in mice reared by $\mathrm{Gabrd}^{-1-}$ mothers. Wild type and $\mathrm{Gabrd}^{-1-}$ mice subjected to unpredictable stress during late pregnancy exhibit decreased pup survival and depression-like behavior in the postpartum period. Exogenous corticosterone treatment in wild type mice during late pregnancy is sufficient to decrease pup survival and induce anxiety-like and depression-like behaviors in the offspring. Further, the abnormal behaviors in juvenile mice reared by $\mathrm{Gabrd}^{-1-}$ mice are alleviated by treatment of the mothers with the corticotropinreleasing hormone (CRH) antagonist, Antalarmin. These studies suggest that hyperresponsiveness of the HPA axis is associated with postpartum depression and may mediate the adverse effects of maternal depression on offspring behavior.
\end{abstract}

\section{Introduction}

Maternal depression has adverse effects on infant behavioral, emotional, and cognitive development [1-7]. Studies investigating the impact of postpartum depression on child development have largely relied on correlative studies in humans. However, investigation into the mechanisms mediating the transmission of negative affect from depressed mother to child has been impeded by the lack of useful animal models. We previously characterized a mouse model with deficits in maternal care which exhibits depression-like behaviors during the postpartum period (Gabrd ${ }^{-1-}$ mice) [8]. Here we utilize this mouse model to investigate the mechanisms underlying the negative impact of maternal depression-like behaviors on offspring development.

Deficits in offspring development associated with maternal depression are correlated with elevated levels of stress hormones in both the mother and the fetus [9]. Treatment of pregnant women with exogenous glucocorticoids results in deficits in child development similar to those related to postpartum depression [10], suggesting that the stress response may mediate the adverse effects of maternal depression on offspring development (for review see [11]). The body's physiological response to stress is mediated by the hypothalamic-pituitary-adrenal (HPA) axis and involves the release of $\mathrm{CRH}$ from the hypothalamus, which triggers the release of adrenocorticotropic hormone (ACTH) from the pituitary, ultimately resulting in glucocorticoid (corticosterone in mice and cortisol in humans) release from the adrenal gland. Although basal levels of corticosterone increase throughout pregnancy and lactation, stress-induced elevations in stress hormones are suppressed (for review see [12]), which is thought to protect the fetus from the negative effects of exposure to high levels of glucocorticoids $[13,14]$. 
Accumulating evidence suggests that dysregulation of the HPA axis plays a role in postpartum depression. The HPA axis may be hyperresponsive in postpartum depression, as indicated by elevated levels of cortisol [15], although these findings are controversial $[16,17]$. More convincing evidence exists for increased levels of CRH [18, 19] and ACTH [18] associated with postpartum depression. Accordingly, it has been suggested that $\mathrm{CRH}$ levels are increased in women with postpartum depression and may even be used as a diagnostic criteria for postpartum depression [19]. Elevated levels of stress hormones in the depressed mother result in elevated levels of stress hormones in the infant [20] and higher levels of stress hormones are associated with decreased maternal care and offspring anxiety [21]. Therefore, it is reasonable to hypothesize that dysregulation of the HPA axis may play a role not only in postpartum depression but also in the negative impact of maternal depression on offspring development. Consistent with this hypothesis administration of exogenous corticosterone to the dams during the postpartum period induced behavioral abnormalities in the offspring [22].

The activity of the HPA axis is governed by CRH neurons in the paraventricular nucleus (PVN) of the hypothalamus (for review see [23]). The activity of these neurons, and thus activity of the HPA axis, is tightly regulated by robust GABAergic inhibition (for review see [24-26]), including tonic GABAergic inhibition mediated by $\delta$ subunitcontaining $\mathrm{GABA}_{\mathrm{A}} \mathrm{Rs}$ [27]. Interestingly, we demonstrated that a mouse model deficient in the $\mathrm{GABA}_{\mathrm{A}} \mathrm{R} \delta$ subunit (Gabrd ${ }^{-/-}$mice) exhibit depression-like behaviors exclusively during the postpartum period and deficits in maternal behaviors [8]. A recent study confirmed these findings demonstrating that $\mathrm{Gabrd}^{-/-}$dams provide fragmented maternal care and their offspring exhibit phenotypes similar to those subjected to early life stress [28].

In this study, we utilized this mouse model (Gabrd ${ }^{-1-}$ mice), which exhibit abnormal/fragmented maternal care and depression-like behavior during the postpartum period $[8,28]$, to investigate the impact of maternal depressionlike behavior on offspring development and the involvement of stress-related steroid hormones. It is well known that maternal depression negatively impacts child development in humans [1-7]. Here we reproduce analogous deficits in offspring development associated with maternal depressionlike behavior in $\mathrm{Gabrd}^{-/-}$dams which can be mimicked with unpredictable stress or exogenous corticosterone administration in wild type mice. Further, blocking CRH signaling with Antalarmin during pregnancy in $\mathrm{Gabrd}^{-/-}$mice prevents the adverse behavioral effects on the juvenile offspring. This study demonstrates the utility of $\mathrm{Gabrd}^{-/-}$mice in investigating the pathophysiological mechanisms of postpartum depression and implicates hyperexcitability of the HPA axis in postpartum depression-like behavior and the negative impact on offspring development.

\section{Materials and Methods}

2.1. Animal Handling and Treatment. Adult (3 months old) C57/Bl6 and $\mathrm{Gabrd}^{-/-}$mice were housed at the University of California, Los Angeles (UCLA), Division of Laboratory Animal Medicine. The animals (4/cage) were housed in clear plastic cages in a temperature- and humidity-controlled environment with a $12 \mathrm{~h}$ light/dark cycle (light on at 6 a.m.) and were maintained on an ad libitum diet of lab chow and water. Animals were handled according to protocols approved by the UCLA, Chancellor's Animal Research Committee (ARC). For the pregnancy experiments, C57/Bl6 and $\mathrm{Gabrd}^{-1-}$ adult female mice were exposed to a male mouse for a single dark cycle. The female was checked for a vaginal plug and placed into a separated home cage. The pregnant female was individually housed with the single litter until the pups were weaned. For the cross-fostering experiments, the mothers were removed from the native litter and swapped with a surrogate (either wild type or $\mathrm{Gabrd}^{-/-}$) immediately following delivery. The mothers were exchanged to minimize the disturbance and handling of the pups. The juvenile offspring remained in the home cage with the mother (or surrogate) and littermates until all behavioral testing was complete.

2.2. Unpredictable Stress Paradigm. This study utilized a stress paradigm devised by another research team, which they termed "chronic ultramild stress" $[29,30]$ and has been shown to elevate corticosterone levels during pregnancy [29, 30]. In the current study, this stress paradigm is referred to as unpredictable stress (US). Wild type and $\mathrm{Gabrd}^{-/-}$mice were subjected to unpredictable stress from D14 to D21 of pregnancy as previously described [29, 30]. Wild type and $\mathrm{Gabrd}^{-/-}$mice at D14 of pregnancy were randomly assigned to two groups: Group 1 (stressed) were subjected to an unpredictable stressor (cage tilt, confinement in a small cage, overnight light exposure for a single night, soiled cage for a single 24-hour period, and difficult access to food) during the dark period for 7 consecutive days until D21 of pregnancy unless parturition occurred before at which time subjection to the stressors was immediately ceased. The stressors were alternated to prevent habituation to a single stressor. The periods of stress were separated by stress-free intervals of at least 12 hours. Group 2 (controls) were maintained in their home cage without subjection to the unpredictable stressors.

2.3. Behavioral Tests. Behavioral tests were performed on wild type and $\mathrm{Gabrd}^{-/-}$dams at $48 \mathrm{hrs}$ postpartum, a time point which has previously been demonstrated to be associated with abnormal postpartum and maternal behaviors in $\mathrm{Gabrd}^{-1-}$ mice [8]. Behavioral tests in juvenile mice began at P21 with the open field test followed by the more stressful forced swim test 24 hrs later. P21 was chosen as the time point to test the impact of maternal behavior on the behavior of the offspring since this time point is prior to weaning and at this time the offspring still share a home cage with the mother.

2.3.1. Depression Assay: Porsolt Forced Swim Test. Depression-like behavior was assessed in wild type and Gabrd ${ }^{-/-}$ dams subjected to unpredictable stress (US) at $48 \mathrm{hrs}$ postpartum and in cross-fostered, CORT, and Antalarmin-treated 
offspring at approximately P21 using the forced swim test as previously described $[8,31]$. Briefly, each mouse was placed individually in a glass cylinder $(21 \mathrm{~cm} \times 12 \mathrm{~cm})$, containing $9 \mathrm{~cm}$ of room temperature water $\left(22-25^{\circ} \mathrm{C}\right)$, in which there is no escape. The latency to immobility and the total duration of immobility throughout the 6 min forced swim test were measured. The mouse was considered to be immobile when it ceased swimming and remained floating motionless, except for infrequent movements of a single hindlimb to maintain being afloat. All tests were videotaped and subsequently analyzed and scored.

2.3.2. Anxiety Assay: Open Field Test. Anxiety-like behavior was assessed in cross-fostered, CORT, and Antalarmintreated juvenile offspring at P21 using the open field test. Animals were tested in the same testing area, under bright light, with no visual cues. The apparatus was cleaned with ethanol and water in between animals to prevent olfactory cues. The mice were placed into the center of the open field, which consists of a plexiglass container $(40 \times 30 \times 40 \mathrm{~cm})$ with a grid of squares $(10 \times 7)$ on the bottom. The amount of time spent in the center $(6 \times 3)$ squares was measured over a single $10 \mathrm{~min}$ testing period. The total number of lines crossed (beam breaks) during the 10 min test was also counted.

2.4. Acute Stress Paradigm. The acute stress paradigm was utilized to measure stress-induced elevations in corticosterone in virgin and postpartum wild type and Gabrd ${ }^{-/-}$ mice. An adapted protocol of the $\mathrm{CO}_{2}$ exposure paradigm [32] in adult female wild type and $\mathrm{Gabrd}^{-/-}$mice was used as an acute stressor. Exposure to $35 \% \mathrm{CO}_{2}$ for $2 \mathrm{~min}$ was used to produce marked increases in circulating corticosterone levels [33]. Virgin and postpartum ( $48 \mathrm{hrs)} \mathrm{wild} \mathrm{type} \mathrm{and}$ $\mathrm{Gabrd}^{-/-}$mice were randomly assigned to two groups: Group 1 (stressed) which were subjected to a single episode of $\mathrm{CO}_{2}$ stress (35\% for $2 \mathrm{~min}$ ) and Group 2 (controls) which were handled in a similar way as Group 1 except they received air instead of air enriched with $\mathrm{CO}_{2}$. All animals were handled similarly in which their home cage was inserted into a larger ventilation box where $\mathrm{CO}_{2}$ (or air in the case of controls) was administered. The animals were allowed to recover for $30 \mathrm{~min}$ prior to blood collection.

2.5. Steroid Hormone Concentration Determination. Blood was collected for corticosterone measurements from wild type and $\mathrm{Gabrd}^{-1-}$ mice $30 \mathrm{~min}$ following acute $\mathrm{CO}_{2}$ stress and compared to controls. Mice were anesthetized with isoflurane before whole blood was collected from experimental groups by retro-orbital bleeding between 12 and 14 hrs. Plasma was immediately isolated by high speed centrifugation and stored at $-20^{\circ}$ until use. Corticosterone levels were measured by enzyme immunoassay according to manufacturer's specifications (Enzo Life Sciences) as described previously [27, 34-36]. Briefly, triplicate $5 \mu \mathrm{L}$ plasma samples were assayed and compared to a standard curve using a spectrophotometer (at $450 \mathrm{~nm}$ ). Intra-assay variability of the corticosterone assay was $7.8 \mathrm{ng} / \mathrm{mL}$ between paired samples and the interassay variability was $4.4 \mathrm{ng} / \mathrm{mL}$ for the same samples between assays.

2.6. Corticosterone Implantation. Wild type mice at day 14 (D14) of pregnancy were briefly anaesthetized with halothane until unresponsive to a foot pinch and were either sham implanted or implanted with a 21-day release $10 \mathrm{mg}$ corticosterone pellet (Innovative Research of America, Sarasota, FL). The hair from the incision site on the back of the neck was clipped and swabbed with ethanol and iodine prior to making the incision. A small $1 \mathrm{~cm}$ incision was made on the back of the neck and a small slow-release pellet (or nothing for sham) was placed underneath the skin using forceps without touching the external area. The incision was then closed with sutures. Pup survival was determined at postnatal day 7 (P7) and offspring behavior was assessed in the juveniles at P21. Two mice per litter were randomly selected for behavioral analysis.

2.7. Antalarmin Treatment. Antalarmin was administered to $\mathrm{Gabrd}^{-/-}$mice from D14 to D21 of pregnancy in the drinking water to minimize the handling of the animals. At day 14 of pregnancy, the normal drinking water was replaced with the Antalarmin solution (10 mg Antalarmin $/ 10 \mu \mathrm{L}$ ethanol/100 $\mathrm{mL}$ drinking water). The animals were maintained on either vehicle $(10 \mu \mathrm{L}$ ethanol/100 $\mathrm{mL}$ drinking water) or Antalarmin until D21 of pregnancy or immediately after parturition (if before D21) at which time the animals were returned to normal drinking water. This treatment strategy was previously shown to block elevations in corticosterone levels $[35,36]$. Pup survival was assessed at P7 and offspring behavior was assessed at P21.

2.8. Statistics. A one-way ANOVA with Tukey's post hoc multiple comparisons test was used to determine statistical significance for comparing more than two experimental groups. Student's $t$-test was used to determine statistical significance between two experimental groups. All statistical tests were carried out using Graphpad 6.0 (Prism).

\section{Results}

3.1. Impact of Maternal Depression-Like Behaviors in Gabrd ${ }^{-1-}$ Mice on Offspring Behavior. Here we utilized Gabrd ${ }^{-1-}$ mice [8] to investigate the impact of maternal depression-like behaviors on offspring behavior. To determine if the maternal behavior, per se, directly mediates the deficits in offspring behavior, we performed cross-fostering experiments. Immediately following delivery, the natural mothers of wild type or $\mathrm{Gabrd}^{-/-}$litters were replaced with a surrogate wild type or Gabrd $d^{-1-}$ mother and the behavior of the cross-fostered animals was then assessed in the juvenile offspring at age P21 (Figure 1(a)). Our data demonstrate that juvenile wild type or $\mathrm{Gabrd}^{-/-}$mice reared by mice exhibiting depressionlike behavior during the postpartum period ( $\mathrm{Gabrd}^{-/-}$mice) exhibit increased anxiety-like behavior in the open field test compared to juvenile wild type or $\mathrm{Gabrd}^{-1-}$ mice reared by 


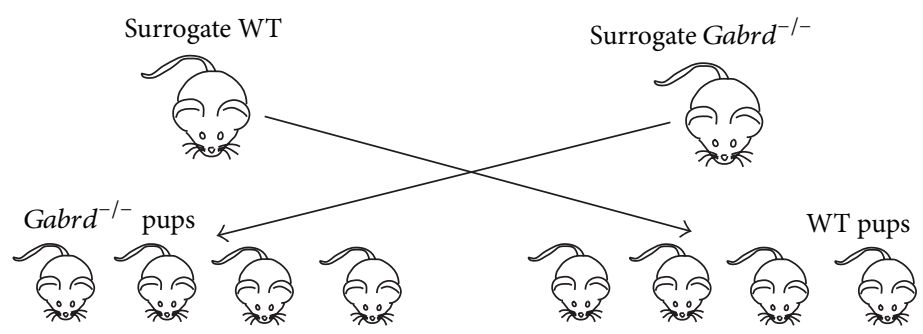

Anxiety- and depression-like behavior assessed in cross-fostered P21 offspring

(a)

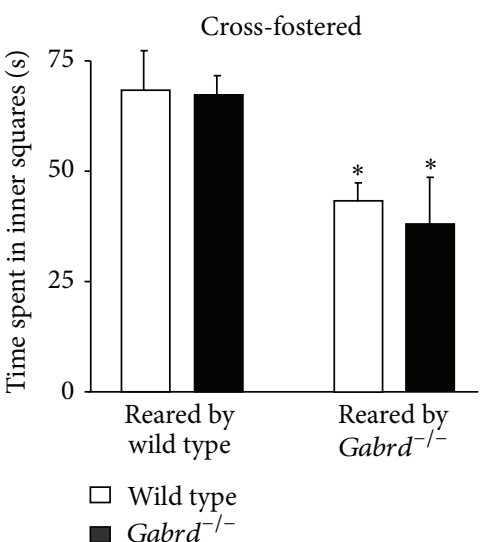

(b)

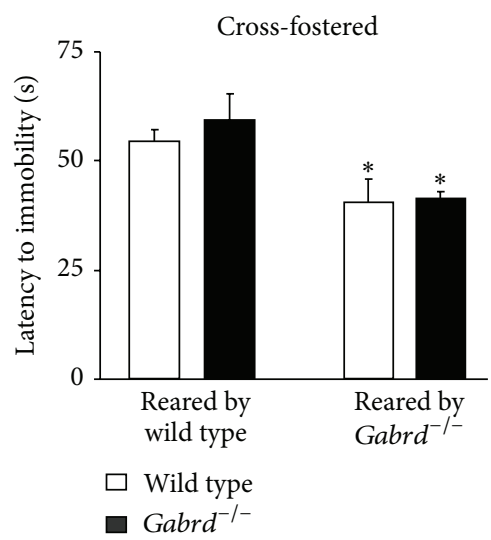

(d)

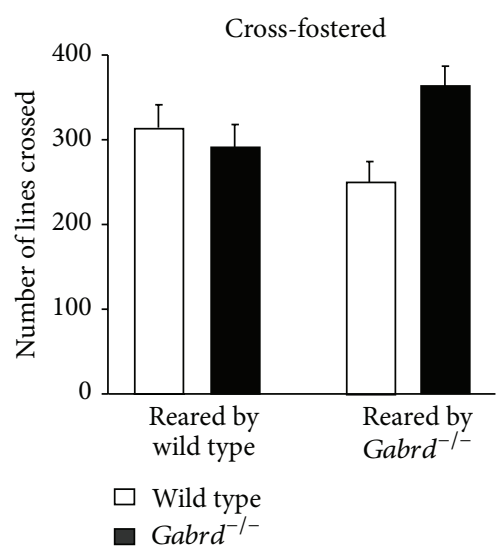

(c)

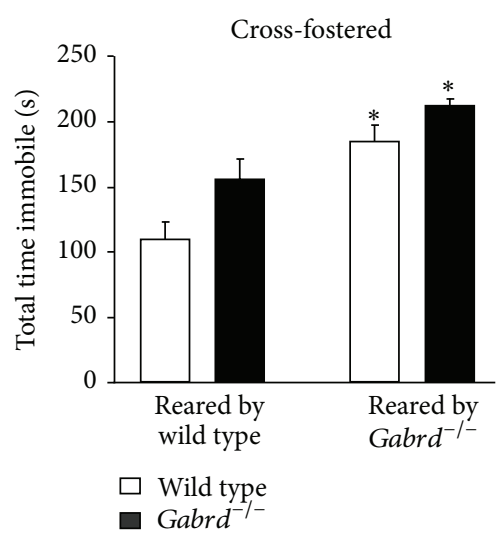

(e)

FiguRE 1: Maternal depression-like behaviors negatively impact offspring behavior. (a) A diagram outlining the experimental design for the cross-fostering experiments. Wild type and $\mathrm{Gabrd}^{-1-}$ mice were reared by either a surrogate wild type or surrogate $\mathrm{Gabrd}^{-/-}$mother. Juvenile wild type and $\mathrm{Gabrd}^{-/-}$offspring reared by surrogate mothers exhibiting depression-like behavior (Gabrd ${ }^{-/-}$mice) exhibit anxietylike behavior in the open field test, evident by a significant decrease in the time spent in the center squares (b) with no change in the number of lines crossed (c) during the 10 min test. $n=12-17$ mice per experimental group; $*$ denotes $p<0.05$ using a one-way ANOVA with Tukey's multiple comparisons test. Juvenile wild type and $\mathrm{Gabrd}^{-/-}$offspring reared by surrogate mothers exhibiting depression-like behavior $\left(\mathrm{Gabrd}^{-1-}\right.$ mice) also exhibit depression-like behavior, assessed using the forced swim test. Offspring reared by surrogate Gabrd $\mathrm{fl}^{-1-}$ mothers exhibit a significant decreased latency to immobility (d) and an increase in the total time spent immobile (e) compared to offspring reared by surrogate wild type mothers. $n=10-12$ mice per experimental group; $*$ denotes $p<0.05$ using one-way ANOVA with Tukey's multiple comparisons test.

surrogate wild type mothers (Table 1; Figure 1(b)). Juvenile mice reared by $\mathrm{Gabrd}^{-1-}$ mice spent less time in the center squares of the open field test compared to juvenile mice reared by wild type mothers (Table 1; Figure $1(\mathrm{~b}))(n=12-17$ mice per experimental group; $*$ denotes $p<0.05$ using a one-way ANOVA with Tukey's multiple comparisons test; $F(3,54)=7.778)$. However, there is no significant difference in the locomotor behavior, indicated by the number of lines crossed, between offspring reared by wild type and $\mathrm{Gabrd}^{-1-}$ mothers (Table 1; Figure 1(c)) $(n=12-17$ mice per 
TABLE 1: Summary of experimental results.

\begin{tabular}{|c|c|c|c|c|c|c|}
\hline & \multirow[b]{2}{*}{ Surrogate } & \multirow{2}{*}{$\begin{array}{c}\text { Pup survival } \\
\%\end{array}$} & \multicolumn{2}{|c|}{ Open field } & \multicolumn{2}{|c|}{ Forced swim test } \\
\hline & & & Time in center $(\mathrm{s})$ & Beam breaks & $\begin{array}{c}\text { Latency to } \\
\text { immobility } \\
\text { (s) }\end{array}$ & $\begin{array}{c}\text { Total time } \\
\text { immobile (s) }\end{array}$ \\
\hline \multicolumn{7}{|l|}{ Dams } \\
\hline Wild type & & $92.0 \pm 1.0$ & & & $70.7 \pm 6.2$ & $148.3 \pm 26.7$ \\
\hline Wild type CUS & & $56.2 \pm 0.9^{*}$ & & & $49.5 \pm 8.2^{*}$ & $245.6 \pm 3.3^{*}$ \\
\hline $\mathrm{Gabrd}^{-/-}$ & & $65.5 \pm 0.9^{*}$ & & & $47.7 \pm 2.2^{*}$ & $205.5 \pm 9.8^{*}$ \\
\hline $\mathrm{Gabrd}^{-1-}$ CUS & & $41.7 \pm 1.1^{*}$ & & & $55.8 \pm 5.2^{*}$ & $222.8 \pm 8.1^{*}$ \\
\hline Wild type sham & & $88.9 \pm 2.7$ & & & & \\
\hline Wild type CORT & & $20.5 \pm 4.4^{*}$ & & & & \\
\hline Gabrd $d^{-1-}$ vehicle & & $51.9 \pm 6.2$ & & & & \\
\hline $\mathrm{Gabrd}^{-/-}$Antalarmin & & $75.0 \pm 5.1^{*}$ & & & & \\
\hline \multicolumn{7}{|l|}{ Offspring (P21) } \\
\hline Cross-fostered wild type & WT surrogate & & $82.9 \pm 12.6$ & $324.1 \pm 29.1$ & $53.6 \pm 2.7$ & $107.1 \pm 13.7$ \\
\hline Cross-fostered Gabrd ${ }^{-/-}$mice & WT surrogate & & $78.6 \pm 10.6$ & $331.5 \pm 32.4$ & $58.5 \pm 6.0$ & $152.9 \pm 15.5$ \\
\hline Cross-fostered wild type & $\begin{array}{l}\text { Gabrd }^{-/-} \\
\text {surrogate }\end{array}$ & & $43.3 \pm 4.3^{* *}$ & $250.5 \pm 22.8$ & $39.9 \pm 5.3^{* *}$ & $181.4 \pm 12.3^{* *}$ \\
\hline Cross-fostered Gabrd ${ }^{-1-}$ mice & $\begin{array}{l}\text { Gabrd }^{-/-} \\
\text {surrogate }\end{array}$ & & $40.7 \pm 4.2^{* *}$ & $360.4 \pm 24.4$ & $40.9 \pm 1.4^{* *}$ & $208.4 \pm 5.0^{* *}$ \\
\hline Wild type sham & & & $65.0 \pm 11.2$ & $338.7 \pm 20.4$ & $53.0 \pm 7.5$ & $124.5 \pm 38.2$ \\
\hline Wild type CORT & & & $37.0 \pm 4.0^{* *}$ & $546.9 \pm 18.1^{* *}$ & $41.5 \pm 4.5$ & $204.4 \pm 11.1^{* *}$ \\
\hline Gabrd $d^{-/-}$vehicle & & & $45.1 \pm 7.1$ & $560.3 \pm 43.9$ & $49.1 \pm 4.1$ & $201.0 \pm 3.3$ \\
\hline Gabrd $^{-/-}$Antalarmin & & & $96.7 \pm 12.8^{* *}$ & $567.0 \pm 65.4$ & $71.0 \pm 9.3^{* *}$ & $100.0 \pm 23.1^{* *}$ \\
\hline
\end{tabular}

* Significance $p<0.05$ compared to control, sham, or vehicle.

${ }^{* *}$ Significance $p<0.05$ compared to wild type, sham, or vehicle.

experimental group; $*$ denotes $p<0.05$ using a one-way ANOVA with Tukey's multiple comparisons test; $F(3,54)=$ 2.819). These results suggest that maternal depression-like behaviors negatively impact offspring development, resulting in increased anxiety-like behavior. In addition, both wild type and $\mathrm{Gabrd}^{-1-}$ juvenile mice, reared by Gabrd ${ }^{-1-}$ mothers, exhibit an increase in depression-like behavior compared to mice reared by surrogate wild type mothers. Juvenile mice reared by Gabrd ${ }^{-1-}$ mice exhibit a decreased latency to immobility and an increase in the total time spent immobile in the forced swim test compared to juvenile mice reared by wild type mothers (Table 1; Figures $1(\mathrm{~d})$ and 1(e)) $(n=$ 10-12 mice per experimental group; $*$ denotes $p<0.05$ using a one-way ANOVA with Tukey's multiple comparisons test; latency: $F(3,39)=4.386$; total time: $F(3,39)=$ 12.61). These data demonstrate that maternal depression-like behaviors in $\mathrm{Gabrd}^{-1-}$ mice during the postpartum period negatively impact offspring development and validate the use of this model for investigating the mechanisms mediating the negative impact of maternal depression on offspring development.

3.2. Role of Stress in Abnormal Maternal and Postpartum Behaviors. We proposed that the abnormal postpartum behaviors in $\mathrm{Gabrd}^{-/-}$mice may be associated with altered stress reactivity during the postpartum period. Postpartum $\mathrm{Gabrd}^{-1-}$ mice exhibit an increase in corticosterone levels following acute $\mathrm{CO}_{2}$ stress $(384.6 \pm 27.6 \mathrm{ng} / \mathrm{mL})$ compared to postpartum wild type mice $(81.0 \pm 10.4 \mathrm{ng} / \mathrm{mL})$, virgin wild type mice $(241.0 \pm 45.3 \mathrm{ng} / \mathrm{mL})$, or virgin $\mathrm{Gabrd}^{-/-}$ mice $(225.6 \pm 32.7 \mathrm{ng} / \mathrm{mL})$ (Figure 2). However, there is no significant difference in circulating corticosterone levels between unstressed postpartum $\mathrm{Gabrd}^{-/-}$mice $(20.5 \pm$ $4.5 \mathrm{ng} / \mathrm{mL})$, postpartum wild type mice $(16.8 \pm 4.1 \mathrm{ng} / \mathrm{mL})$, virgin $\mathrm{Gabrd}^{-/-}$mice $(59.9 \pm 10.6 \mathrm{ng} / \mathrm{mL})$, or virgin wild type mice $(30.7 \pm 5.3 \mathrm{ng} / \mathrm{mL})$ (Figure 2$)(n=7-17$ mice per experimental group; $*$ denotes $p<0.05$ using a one-way ANOVA with Tukey's multiple comparisons test; $F(8,93)=$ 20.94).

If altered stress reactivity plays a role in maternal depression-like behaviors in Gabrd ${ }^{-l-}$ mice, then we hypothesized that chronic stress would be sufficient to induce the same behavioral disturbances in wild type mice during the postpartum period. Wild type mice subjected to unpredictable stress (US) from D14 to D21 of pregnancy (Figure 3(a)) exhibit a decrease in the survival rate of their pups compared to unstressed controls (Table 1; Figure 3(b)) ( $n$ : wild type $=12$ mothers, 100 pups; wild type US $=9$ mothers, 73 pups; $*$ denotes $p<0.05$ using Student's $t$ test). Decreased pup survival is exacerbated in $\mathrm{Gabrd}^{-1-}$ 


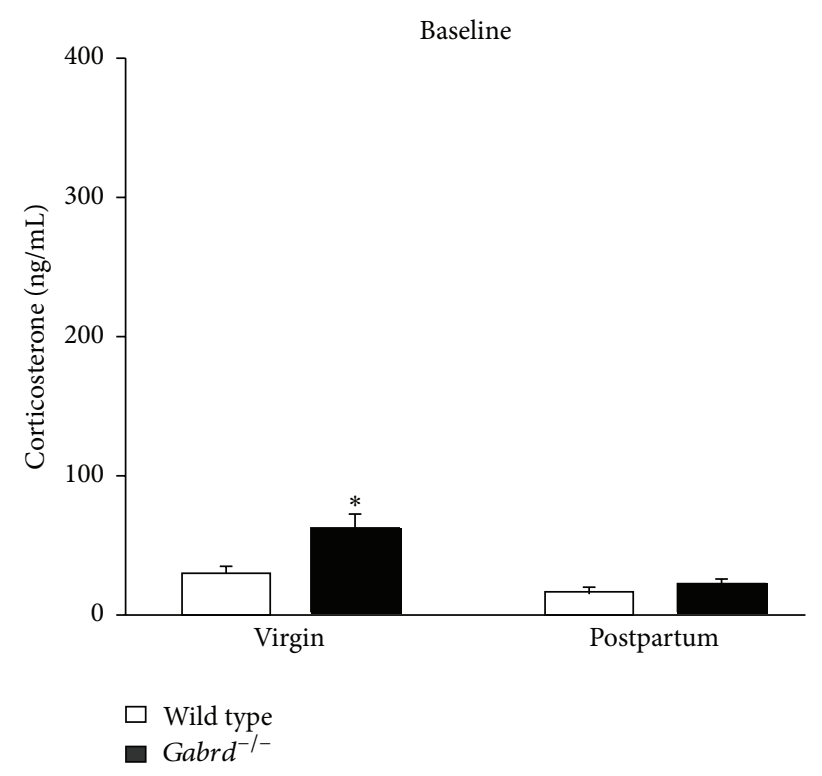

(a)

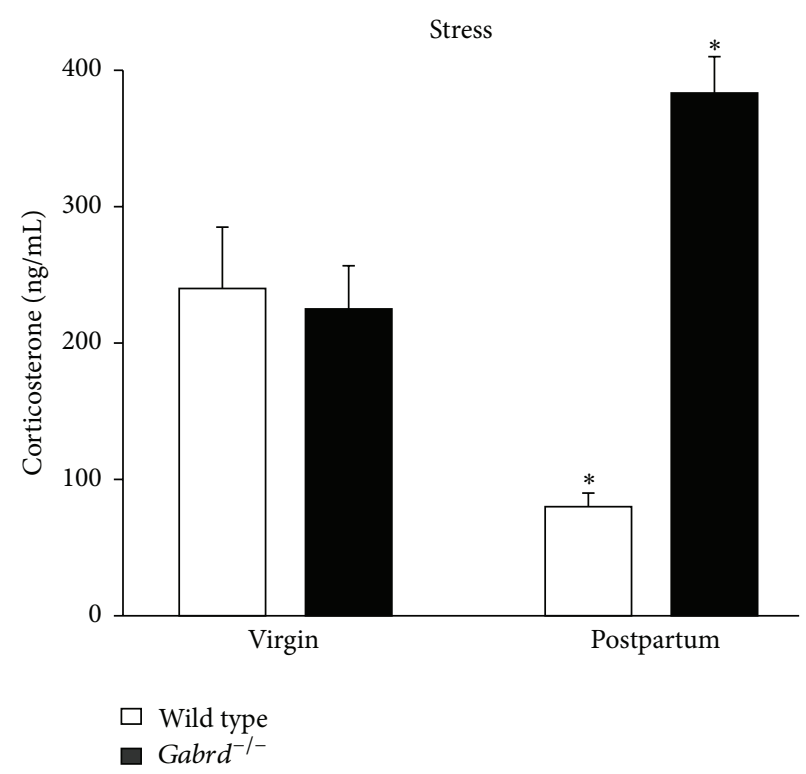

(b)

FIGURE 2: HPA axis hyperexcitability in postpartum $\mathrm{Gabrd}^{-1-}$ mice. (a) Baseline corticosterone levels measured in the plasma of virgin and postpartum wild type and $\mathrm{Gabrd}^{-1-}$ mice. (b) Average circulating corticosterone levels following $\mathrm{CO}_{2}$ stress in virgin and postpartum wild type and $\mathrm{Gabrd}^{-1-}$ mice. ( $n=7-17$ mice per experimental group; $*$ denotes $p<0.05$ using one-way ANOVA with Tukey's multiple comparisons test).

mice subjected to US compared to unstressed $\mathrm{Gabrd}^{-/-}$mice (Table 1; Figure 3(b)) (n: Gabrd ${ }^{-/-}=12$ mothers, 84 pups; Gabrd $^{-1-}$ US: 5 mothers, 36 pups; * denotes $p<0.05$ using Student's $t$-test). Dams subjected to US also fail to build a nest and keep the pups at an increased distance from the mother (data not shown), similar to $\mathrm{Gabrd}^{-/-}$mice [8]. These abnormal maternal behaviors in wild type mice subjected to the US paradigm are associated with depressionlike behaviors in the dams (Figures 3(c)-3(d)). Wild type mice subjected to unpredictable stress exhibit a decreased latency to immobility and an increased total time spent immobile in the forced swim test at 48 hours postpartum compared to unstressed postpartum wild type mice (Table 1; Figures 3(c)-3(d)) ( $n=5-9$ for each experimental group; $*$ denotes $p<0.05$ using a one-way ANOVA with Tukey's multiple comparisons test; latency: $F(3,22)=2.729$; total time: $F(3,22)=7.874)$. These data are consistent with the hypothesis that altered stress reactivity plays a role in mediating abnormal postpartum behaviors.

\subsection{Role of Maternal Corticosterone on Offspring Behavior.} To investigate whether altered stress reactivity in $\mathrm{Gabrd}^{-/-}$ mice plays a role in mediating the negative impact of maternal depression-like behaviors on offspring development, we either sham-implanted wild type mice or implanted them with a slow-release $10 \mathrm{mg}$ corticosterone pellet on day 14 of pregnancy and assessed offspring behavior at P21 (Figure 4(a)). We determined that corticosterone treatment does not interfere with pup delivery or litter size $(7.3 \pm$ 0.9 pups) compared to sham implanted mice $(6.0 \pm 0.8$ pups) ( $n$ : sham $=9$ mothers, 54 pups; CORT: 10 mothers,
73 pups; $*$ denotes $p<0.05$ using Student's $t$-test). Note: litter sizes were determined at the time of delivery since there is a decreased survival rate of the pups born to corticosterone implanted mice (Figure 4(b)); however, all pups were alive at the time of delivery. Corticosterone levels are significantly elevated in the corticosterone implanted dams at 48 hours postpartum $(192.8 \pm 50.3 \mathrm{ng} / \mathrm{mL})$ compared to sham implanted mice $(29.8 \pm 3.2 \mathrm{ng} / \mathrm{mL})$, postpartum wild type controls $(16.8 \pm 4.1 \mathrm{ng} / \mathrm{mL})$, or stressed postpartum wild type mice $(81.0 \pm 10.4 \mathrm{ng} / \mathrm{mL})(n=10-15$ mice per experimental group; $*$ denotes $p<0.05$ using Student's $t$ test). Corticosterone treatment in the mothers at D14 was sufficient to induce abnormal postpartum behaviors in wild type mice, such as inability to build a proper nest (data not shown) and an increase in pup mortality rate due to cannibalism or neglect (Table 1; Figure 4(b)), similar to that seen in $\mathrm{Gabrd}^{-/-}$mice [8] ( $n$ : sham $=9$ mothers, 54 pups; CORT: 10 mothers, 73 pups; $*$ denotes $p<0.05$ using Student's $t$-test). Corticosterone treatment in the mother was also sufficient to induce behavioral deficits in their juvenile offspring. Juvenile mice (P21) reared by mothers treated with corticosterone spent less time in the center squares of the open field test compared to juvenile mice reared by sham implanted wild type mothers (Table 1; Figure $4(\mathrm{c}))(n=8$ mice per experimental group, 2 mice per litter in 4 different litters; $*$ denotes $p<0.05$ using Student's $t$-test), indicative of anxiety-like behavior. In addition, corticosterone treatment alters locomotor behavior in the offspring of corticosteronetreated mothers, evident from the increased number of lines crossed in the open field test compared to sham implanted mothers (Table 1; Figure $4(\mathrm{c}))(n=8$ mice per experimental 


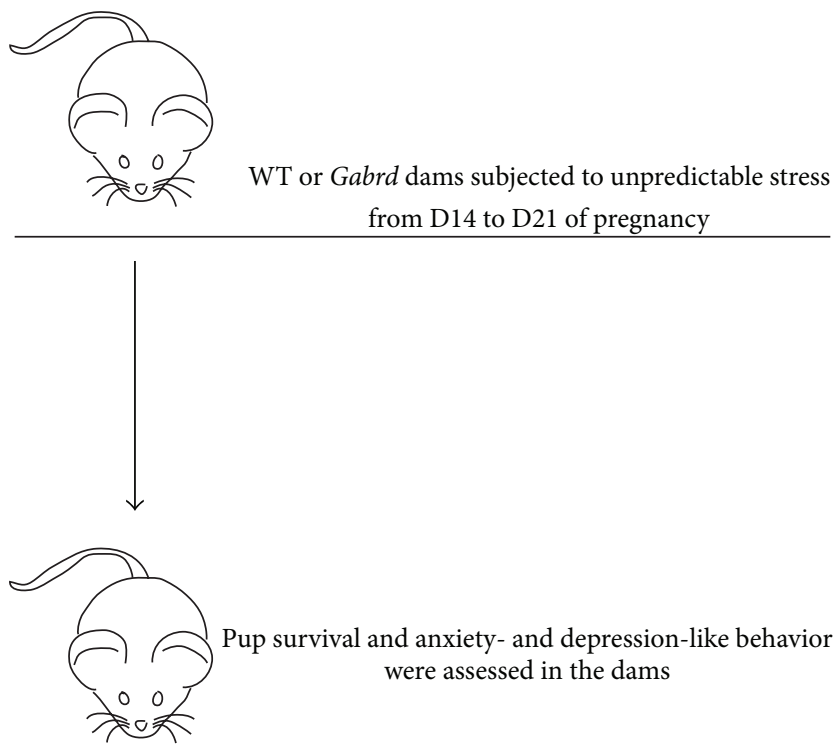

(a)

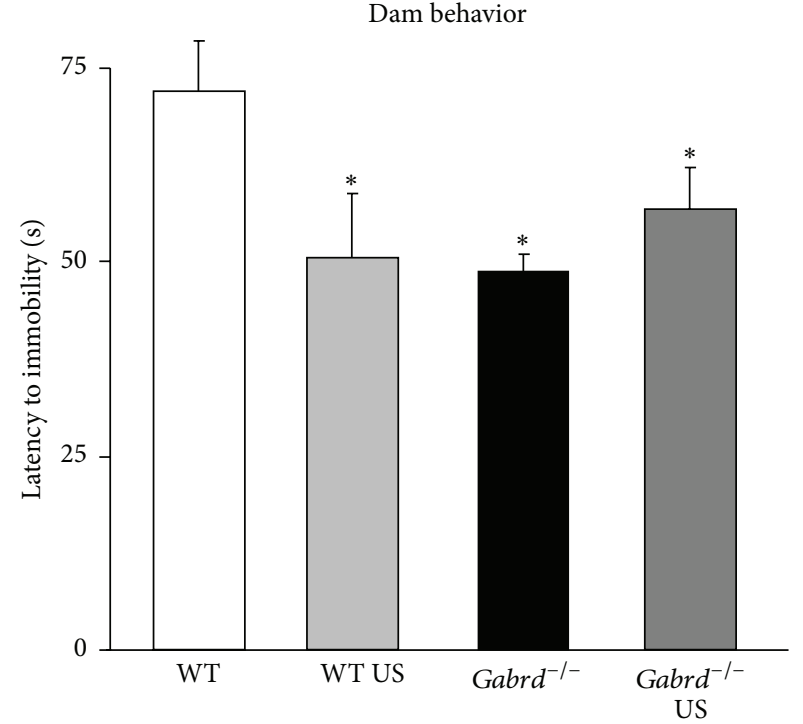

(c)

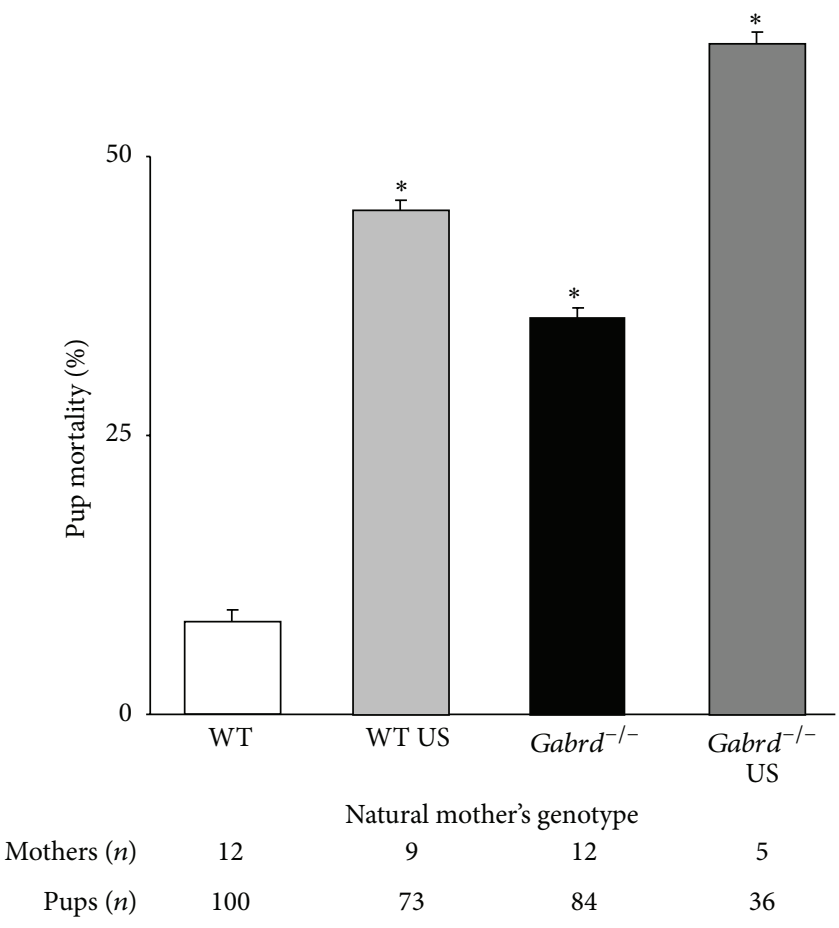

(b)

Dam behavior

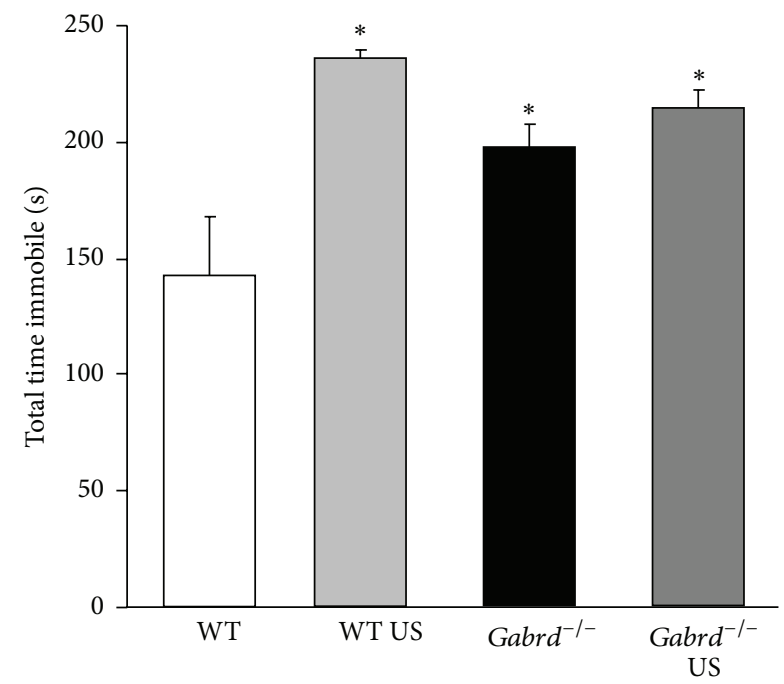

(d)

FIGURE 3: Unpredictable stress during pregnancy results in decreased pup survival and depression-like behavior in the dams. (a) A diagram outlining the experimental design. Wild type and $\mathrm{Gabrd}^{-/-}$dams were subjected to unpredictable stress from D14 to D21 of pregnancy and pup survival and depression-like behavior were assessed in the dams at $48 \mathrm{hrs}$ postpartum. (b) Wild type and Gabrd ${ }^{-/-}$mice subjected to unpredictable stress from D14 to D21 of pregnancy exhibit a decrease in pup survival compared to control wild type and Gabrd ${ }^{-/-}$mice. $n$ : wild type $=12$ mothers, 100 pups; wild type US $=9$ mothers, 73 pups; Gabrd ${ }^{-/-}=12$ mothers, 84 pups; Gabrd ${ }^{-/-}$US $=5$ mothers, 36 pups; * denotes $p<0.05$ using Student's $t$-test. Wild type and $\mathrm{Gabrd}^{-/-}$dams subjected to unpredictable stress from D14 to D21 of pregnancy exhibit a decreased latency to immobility (c) and an increased total time spent immobile (d) in the forced swim test at 48 hrs postpartum. $n=5-9$ for each experimental group; $*$ denotes $p<0.05$ using one-way ANOVA with Tukey's multiple comparisons test. 


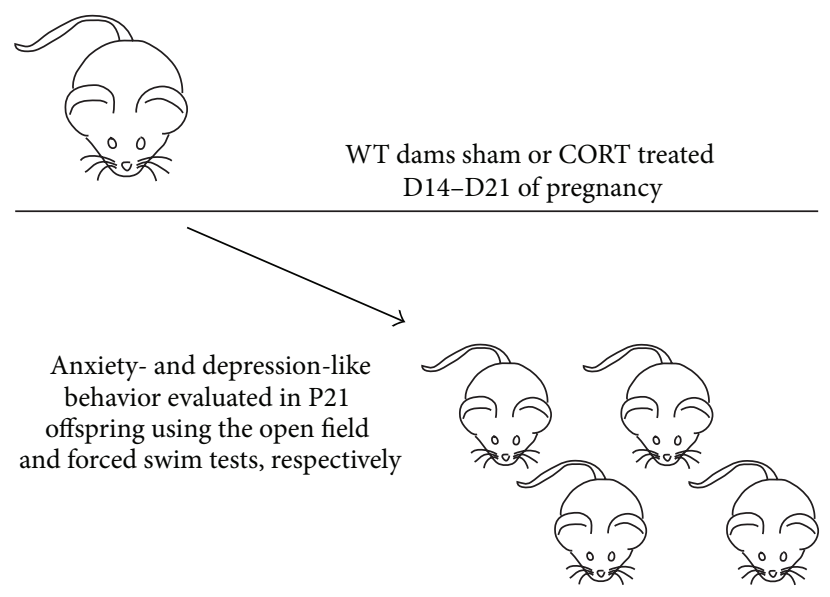

(a)

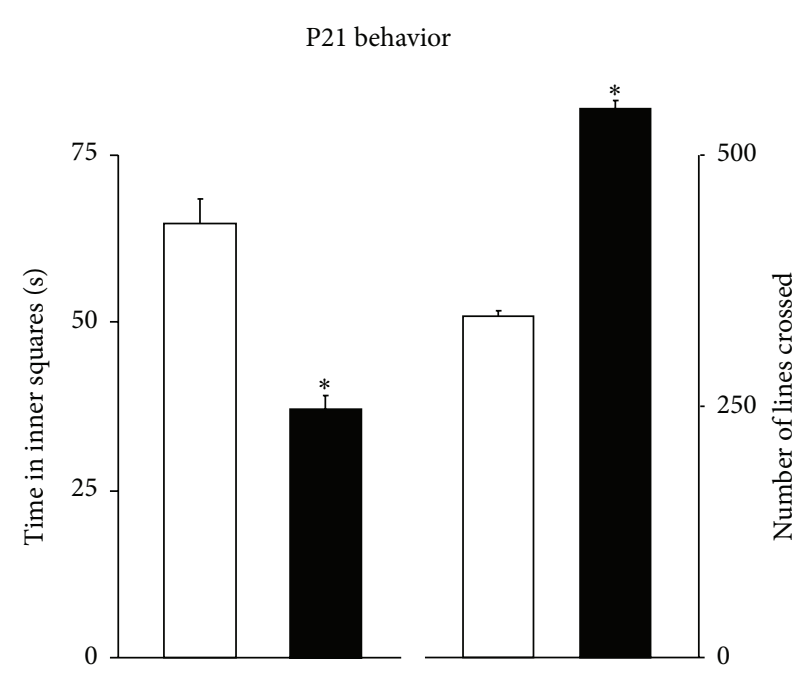

(c)

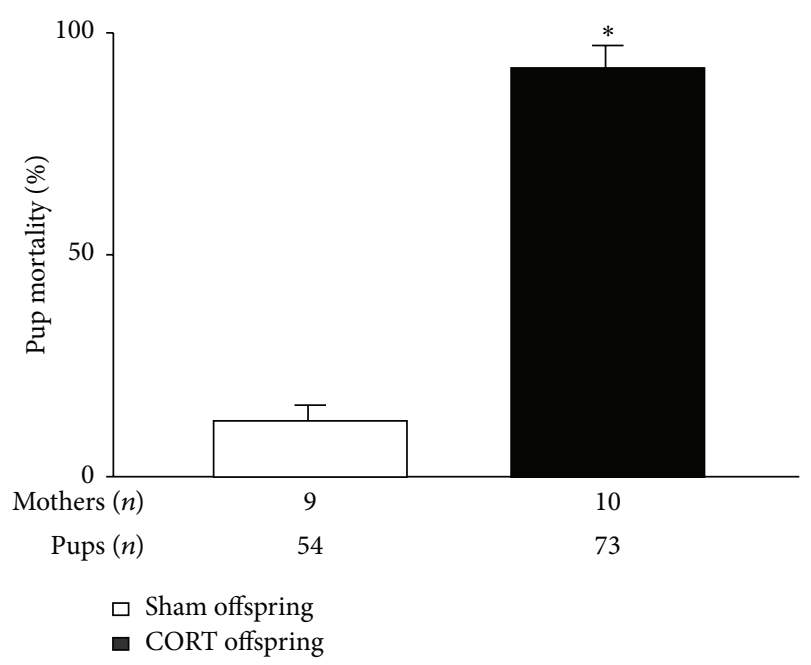

(b)

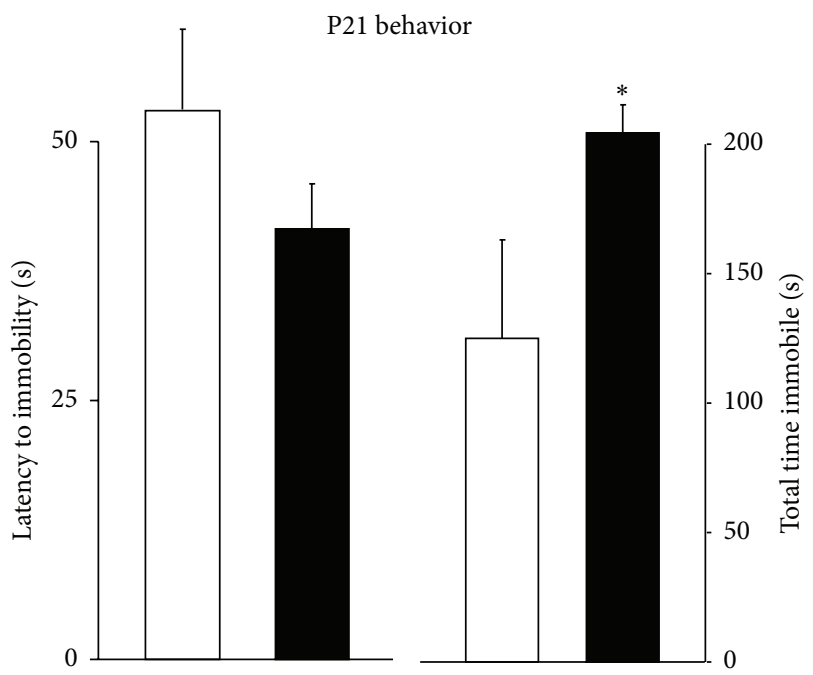

(d)

FIGURE 4: Corticosterone treatment in wild type mice during pregnancy decreases pup survival and induces deficits in offspring behavior. (a) A diagram outlining the experimental design. Wild type dams were implanted with a $10 \mathrm{mg}$, slow-release corticosterone pellet at D14 of pregnancy and pup survival and offspring behavior in the juveniles were assessed at P21. (b) Corticosterone-treated wild type mice exhibit a decrease in pup survival compared to sham implanted wild type mice. $n$ : sham $=9$ mothers, 54 pups; CORT: 10 mothers, 73 pups; $*$ denotes $p<0.05$ using Student's $t$-test. (c-d) Juvenile offspring reared by wild type mothers implanted with a $10 \mathrm{mg}$, slow-release corticosterone pellet at D14 of pregnancy, exhibit anxiety-like and depression-like behaviors at P21. (c) Juvenile mice reared by corticosterone-treated mothers spent a decreased amount of time in the center of the open field test and an increase in locomotor activity. (d) Juvenile mice reared by corticosterone-treated mothers exhibited a decreased latency to immobility and an increase in the total time spent immobile in the forced swim test ( $n=8$ mice per experimental group; $*$ denotes $p<0.05$ using Student's $t$-test).

group, 2 mice per litter in 4 different litters; * denotes $p<0.05$ using Student's $t$-test). Corticosterone treatment in wild type mothers also induced depression-like behavior in the offspring. Offspring reared by corticosterone implanted wild type mothers spend an increased total time immobile during the forced swim test compared to juvenile mice reared by sham implanted wild type mothers (Table 1; Figure $4(\mathrm{~d})$ ) ( $n=8$ mice per experimental group, 2 mice per litter in 4 different litters; $*$ denotes $p<0.05$ using Student's $t$ test). These data support the hypothesis that stress hormones, specifically corticosterone, mediate the negative impact of maternal depression-like behaviors on offspring behavior in the mouse.

If altered stress reactivity in $\mathrm{Gabrd}^{-/-}$mothers plays a role in the impact of maternal depression-like behaviors on offspring development, we hypothesized that inhibiting the stress response in the mother with the corticotropinreleasing hormone $(\mathrm{CRH})$ antagonist, Antalarmin, would decrease the anxiety- and depression-like behaviors in the juvenile offspring (Figure 5(a)). We did not observe any changes in litter size associated with Antalarmin treatment (7.6 \pm 0.9 pups) compared to vehicle treatment $(6.5 \pm 0.8$ 


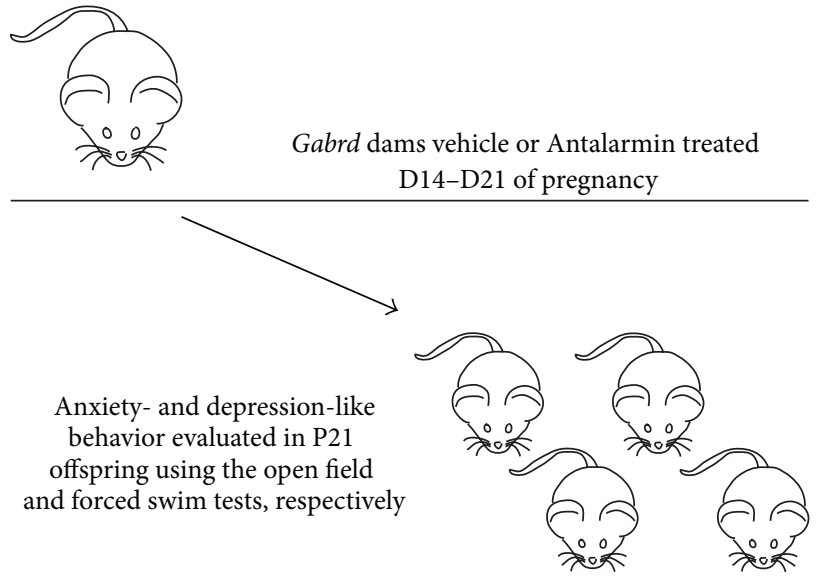

(a)

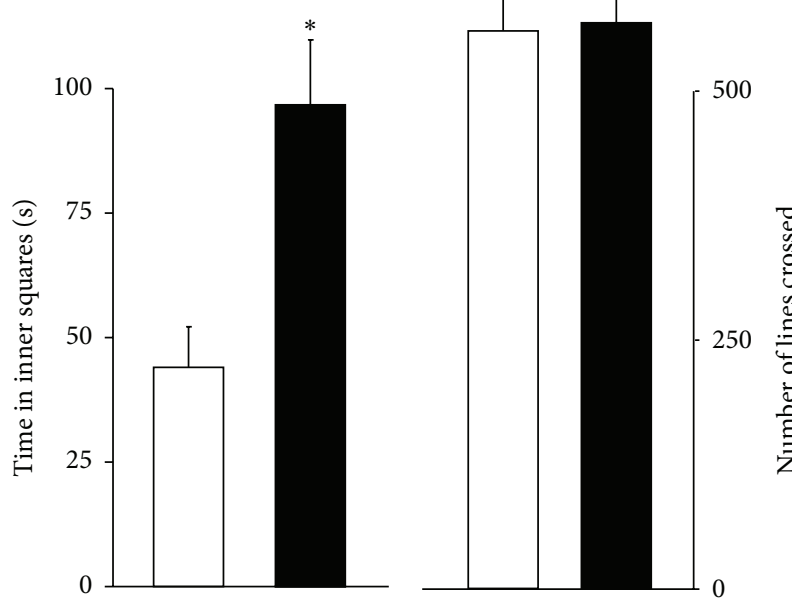

(c)

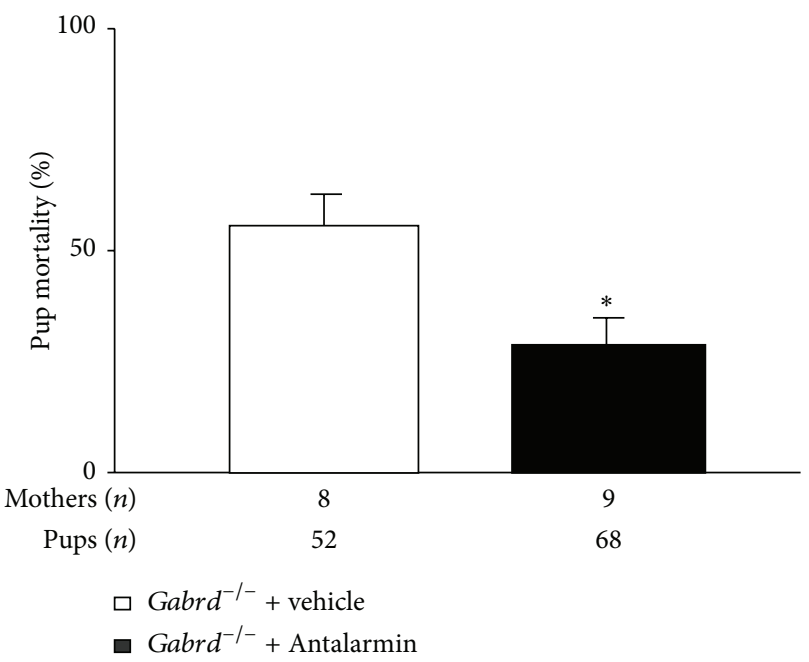

(b)

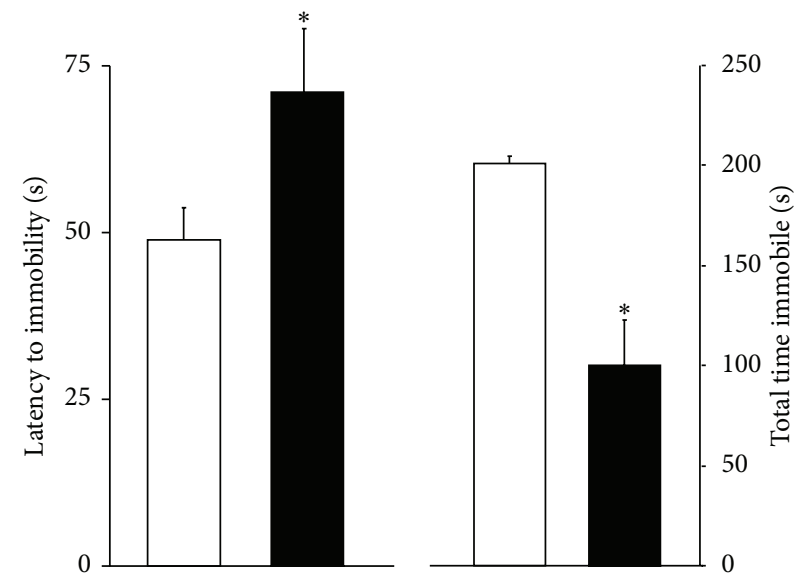

(d)

FIGURE 5: Blocking CRH signaling in $\mathrm{Gabrd}^{-1-}$ mice during pregnancy increased pup survival and diminished the negative impact on offspring behavior. (a) A diagram outlining the experimental design. Gabrd ${ }^{-/}$dams were treated with Antalarmin from D14 to D21 of pregnancy and pup survival and offspring behavior in the juveniles were assessed at P21. (b) Antalarmin-treated Gabrd ${ }^{-1-}$ mothers exhibit an increase in pup survival compared to vehicle-treated controls. $n$ : Gabrd ${ }^{-1-}$ vehicle $=8$ mothers, 52 pups; Gabrd $d^{-1-}+$ Antalarmin: 9 mothers, 68 pups; significance was determined as $p<0.05$ using Student's $t$-test. (c-d) Juvenile mice reared by Gabrd ${ }^{-/-}$mothers treated with Antalarmin exhibit a decrease in anxiety-like and depression-like behavior at P21. (c) Offspring of Antalarmin-treated Gabrd ${ }^{-/-}$mothers spend an increased amount of time in the center of the open field with no change in activity compared to vehicle-treated controls. (d) Offspring of Antalarmin-treated $\mathrm{Gabrd}^{-/-}$mothers exhibited an increased latency to immobility and a decreased total time spent immobile in the forced swim test ( $n=8-10$ mice per experimental group; $*$ denotes $p<0.05$ using Student's $t$-test).

pups). This dose of Antalarmin was sufficient to decrease the stress-induced circulating corticosterone levels in postpartum $\mathrm{Gabrd}^{-/-}$mice $(101.5 \pm 12.0 \mathrm{ng} / \mathrm{mL})$ to levels similar to postpartum wild type mice $(81.0 \pm 10.4 \mathrm{ng} / \mathrm{mL})$ which is significantly lower than the stress-induced levels in postpartum $\mathrm{Gabrd}^{-1-}$ mice $(384.6 \pm 27.6 \mathrm{ng} / \mathrm{mL})$. Antalarmin treatment ameliorated the abnormal postpartum behaviors in postpartum Gabrd Gi- $^{-1}$ mice. Gabrd ${ }^{-/-}$dams treated with Antalarmin exhibit an increase in pup survival compared to controls (Table 1; Figure 5(b)) ( $n$ : $\mathrm{Gabrd}^{-/-}$vehicle $=8$ mothers, 52 pups; Gabrd ${ }^{-/-}+$Antalarmin: 9 mothers, 68 pups; significance was determined as $p<0.05$ using Student's $t$-test). Further, Antalarmin treatment in the mother was also sufficient to ameliorate the mood disorders in juvenile mice reared by $\mathrm{Gabrd}^{-1-}$ mothers. Juvenile mice (P21) reared by $\mathrm{Gabrd}^{-/-}$mothers treated with Antalarmin spent more time in the center squares of the open field test, which is indicative of decreased anxiety levels, compared to juvenile mice reared by vehicle-treated $\mathrm{Gabrd}^{-/-}$mothers (Table 1; Figure 5(c)) ( $n=8-10$ offspring per experimental group, 
2 mice per litter in 4-5 different litters; significance was determined as $p<0.05$ using Student's $t$-test). There was no significant difference in the number of lines crossed in the open field test between the offspring of Antalarmin-treated and vehicle-treated Gabrd ${ }^{-/-}$mothers (Table 1; Figure 5(c)). Similarly, offspring reared by Antalarmin-treated $\mathrm{Gabrd}^{-/-}$ mothers exhibit decreased depression-like behavior, evident by an increased latency to immobility and decreased total time spent immobile compared to offspring reared by vehicletreated $\mathrm{Gabrd}^{-/-}$mothers (Table 1; Figure 5(d)) $(n=8-10$ mice per experimental group, 2 mice per litter in 4-5 different litters; significance was determined as $p<0.05$ using Student's $t$-test). These data demonstrate that inhibiting the stress response, such as with the $\mathrm{CRH}$ antagonist, Antalarmin, is therapeutic in ameliorating the abnormal postpartum behaviors in $\mathrm{Gabrd}^{-/-}$mothers as well as preventing the negative impact of maternal depression-like behaviors on offspring development.

\section{Discussion}

This study highlights the utility of a unique mouse model to investigate the underlying mechanisms mediating the pathophysiology of postpartum depression and the mechanism(s) through which postpartum depression negatively impacts offspring development. It is generally accepted that both genetic and environmental factors play a role in the pathophysiology of postpartum depression. However, either the current animal models exhibit a genetic predisposition for depression-like behavior or the behavior is environmentally induced [37]. Here we describe a genetic mouse model exhibiting depression-like behavior that is restricted to the postpartum period [8], which is aggravated by environmental stress similar to the human condition [38]. This is the first genetic mouse model which exhibits a predisposition to postpartum depression-like behavior in which there is also an environmental component. Therefore, we feel that this is a useful model for studying the mechanisms mediating postpartum depression-like behavior and the accompanying deficits in offspring development.

Clearly, hormone changes throughout pregnancy and the postpartum period trigger the onset of postpartum depression. However, gonadal hormone levels do not appear to be significantly altered in women with postpartum depression [39-41], suggesting that women must be predisposed to the disorder. During pregnancy, levels of estrogen and progesterone steadily increase due to placental production of these hormones, which decrease abruptly with the removal of the placenta. However, no change in estrogen or progesterone levels has consistently been shown to be associated with postpartum depression [41]. There are numerous other hormonal changes that occur during pregnancy, including changes in oxytocin, prolactin, and cortisol levels (for review see [42]). However, no alterations out of the physiological range were found for prolactin [41, 43], oxytocin, or vasopressin [44] associated with postpartum depression. Hypercortisolism has been suggested to play a role in the pathophysiology of postpartum depression [45], since major depression is also associated with hypercortisolism [46]. Normally, the stress-induced activation of the HPA axis is suppressed during pregnancy ([47], for review see [12]), consistent with our observations in postpartum wild type mice (Figure 2). Altered levels of cortisol [11, 18, 48], ACTH [18], and $\mathrm{CRH}$ [19] have been associated with postpartum depression. Researchers have gone so far as to say that elevated $\mathrm{CRH}$ levels may be used as a diagnostic criterion for postpartum depression [19]. However, other studies have failed to reproduce these results $([41,43]$, for review see [42]). Here we demonstrate hyperresponsivity of the HPA axis associated with depression-like behaviors during the postpartum period in a mouse model, similar to what has been observed in women with postpartum depression [49].

Consistent with a role for HPA axis hyperresponsiveness in postpartum depression-like behaviors, this study supports a role for elevated corticosterone in the pathophysiology of postpartum mood disorders, since physiological stress is sufficient to induce depression-like behavior during the postpartum period in mice (Figure 3). Previous studies have demonstrated that corticosterone alters maternal care and induces postpartum depression-like behaviors in the dams [50]. Here we demonstrate that exogenous corticosterone treatment in wild type mice during pregnancy results in a robust decrease in pup survival (Figure 4). This has previously been observed [51], albeit not to the same extent as in the current study. The discrepancy may be due to a prolonged exposure in our study to levels of corticosterone normally found in stress. However, we cannot rule out potential abnormalities in the pups due to corticosterone exposure which may impact pup mortality. Interestingly, our study demonstrates that physiological stress in the dams is sufficient to increase pup mortality in both wild type and $\mathrm{Gabrd}^{-/-}$mice (Figure 3(b)). However, unpredictable stress does not alter depression-like behaviors in postpartum $\mathrm{Gabrd}^{-/-}$although it increases depression-like behaviors in wild type mice (Figures 3(c) and 3(d)). We interpret these data to indicate that physiological stress is incapable of altering depression-like behaviors in postpartum $\mathrm{Gabrd}^{-/-}$ mice in which corticosterone levels are already elevated, demonstrating a potential ceiling effect. Similarly, our results demonstrate that the $\mathrm{CRH}$ antagonist, Antalarmin, increases pup survival in Gabrd ${ }^{-/-}$mice (Figure 5). These data demonstrate a direct role of stress hormones in postpartum mood disorders and may contribute to the negative impact of maternal depression on offspring development.

Children exposed to mothers with postpartum depression exhibit deficits in cognitive development, motor, and emotional development (for review see [52]). Many mechanisms have been proposed to mediate the negative association between maternal depression and offspring development, including environmental and genetic components. It is also possible that there is a direct, biochemical component of maternal depression which impacts offspring development. Deficits in child development associated with maternal depression are correlated with elevated cortisol levels in the mother $[53,54]$, suggesting that the stress hormones may play a role in the negative impact of maternal depression on child 
development. Consistent with this theory, we demonstrate behavioral deficits in mice reared by $\mathrm{Gabrd}^{-1-}$ mothers, which exhibit hyperresponsiveness of the HPA axis, and offspring reared by wild type mice subjected to US or treated with exogenous corticosterone. Further, corticosterone treatment in dams has previously been shown to result in adverse behavioral effects in the offspring [22], which could be a direct effect of corticosterone levels in the offspring which may alter subsequent HPA axis activity [55]. These data support a direct role of stress hormones in both the pathophysiology of postpartum depression and the negative impact of maternal depression on offspring development.

In the current study, the mouse models which exhibit abnormal postpartum behaviors and a negative impact offspring development, including $\mathrm{Gabrd}^{-1-}$ mice and wild type dams subjected to unpredictable stress or treated with exogenous corticosterone, exhibit a high degree of pup mortality due to cannibalism and/or neglect. This is in contrast to the human condition of postpartum depression which is not typically associated with infant mortality or infanticide. Neonaticide and infanticide are more commonly associated with postpartum psychosis [56]. It is possible that postpartum $\mathrm{Gabrd}^{-/-}$mice more accurately model postpartum psychosis. However, this is difficult to assess in mice and requires further study.

Due to the high level of pup mortality in the mouse models exhibiting abnormal postpartum behaviors and a negative impact offspring development, including $\mathrm{Gabrd}^{-/-}$ mice and wild type dams subjected to unpredictable stress or treated with exogenous corticosterone, we cannot rule out both the impact of changing litter size on maternal behaviors and the impact on offspring development. Relevant to the current study, smaller litter size is associated with more directed maternal care $[57,58]$. Further, mice reared in smaller litters also exhibit decreased anxiety-like behaviors during adulthood [58]. These findings are in contrast to the current study where we observe a decreased litter size in $\mathrm{Gabrd}^{-/-}$litters associated with abnormal maternal care [8] and increased anxiety- and depression-like behaviors in the offspring reared by $\mathrm{Gabrd}^{-1-}$ mice (Figure 1). Thus, it does not appear that litter size impacts the findings in the current study.

This study directly demonstrates the negative impact of postpartum depression-like behavior and deficits in maternal care on offspring behavior. Further, our data suggest a role for HPA axis dysfunction in mediating the negative impact of maternal mood on offspring behavior.

\section{Conflict of Interests}

The authors declare that there is no conflict of interests regarding the publication of this paper.

\section{Acknowledgments}

This work was supported by Grants from the NIH/NIMH MH076994 (Istvan Mody), NIH/NINDS 073574 (Jamie Maguire), and the Charles H. Hood Foundation (Jamie
Maguire). The investigators would like to thank Reyes Main Lazaro for his expertise in the timed pregnancies and the blood sample collections. His assistance on this project was greatly appreciated. The authors would also like to acknowledge the technical contribution of Isabella Ferando and Charlotte Simonsen on this study.

\section{References}

[1] R. Feldman, A. Granat, C. Pariente, H. Kanety, J. Kuint, and E. Gilboa-Schechtman, "Maternal depression and anxiety across the postpartum year and infant social engagement, fear regulation, and stress reactivity," Journal of the American Academy of Child and Adolescent Psychiatry, vol. 48, no. 9, pp. 919-927, 2009.

[2] S. L. Grace, A. Evindar, and D. E. Stewart, "The effect of postpartum depression on child cognitive development and behavior: a review and critical analysis of the literature," Archives of Women's Mental Health, vol. 6, no. 4, pp. 263-274, 2003.

[3] K. Lyons-Ruth, D. Zoll, D. Connell, and H. U. Grunebaum, "The depressed mother and her one-year-old infant: environment, interaction, attachment, and infant development," New Directions for Child Development, no. 34, pp. 61-82, 1986.

[4] L. Murray, "The impact of postnatal depression on infant development," Journal of Child Psychology and Psychiatry and Allied Disciplines, vol. 33, no. 3, pp. 543-561, 1992.

[5] L. Murray, A. Fiori-Cowley, R. Hooper, and P. Cooper, "The impact of postnatal depression and associated adversity on early mother-infant interactions and later infant outcome," Child Development, vol. 67, no. 5, pp. 2512-2526, 1996.

[6] L. Murray, D. Sinclair, P. Cooper, P. Ducournau, P. Turner, and A. Stein, "The socioemotional development of 5-year-old children of postnatally depressed mothers," Journal of Child Psychology and Psychiatry, vol. 40, no. 8, pp. 1259-1271, 1999.

[7] M. Righetti-Veltema, A. Bousquet, and J. Manzano, "Impact of postpartum depressive symptoms on mother and her 18month-old infant," European Child and Adolescent Psychiatry, vol. 12, no. 2, pp. 75-83, 2003.

[8] J. Maguire and I. Mody, “GABA $\mathrm{A}_{\mathrm{A}} \mathrm{R}$ plasticity during pregnancy: relevance to postpartum depression," Neuron, vol. 59, no. 2, pp. 207-213, 2008.

[9] R. Gitau, A. Cameron, N. M. Fisk, and V. Glover, "Fetal exposure to maternal cortisol," The Lancet, vol. 352, no. 9129, pp. 707-708, 1998.

[10] D. Owen, M. H. Andrews, and S. G. Matthews, "Maternal adversity, glucocorticoids and programming of neuroendocrine function and behaviour," Neuroscience \& Biobehavioral Reviews, vol. 29, pp. 209-226, 2005.

[11] M. Bloch, R. C. Daly, and D. R. Rubinow, "Endocrine factors in the etiology of postpartum depression," Comprehensive Psychiatry, vol. 44, no. 3, pp. 234-246, 2003.

[12] D. A. Slattery and I. D. Neumann, "No stress please! Mechanisms of stress hyporesponsiveness of the maternal brain," The Journal of Physiology, vol. 586, no. 2, pp. 377-385, 2008.

[13] P. J. Brunton and J. A. Russell, "Attenuated hypothalamopituitary-adrenal axis responses to immune challenge during pregnancy: the neurosteroid-opioid connection," Journal of Physiology, vol. 586, no. 2, pp. 369-375, 2008.

[14] P. J. Brunton, J. A. Russell, and A. J. Douglas, "Adaptive responses of the maternal hypothalamic-pituitary-adrenal axis 
during pregnancy and lactation," Journal of Neuroendocrinology, vol. 20, no. 6, pp. 764-776, 2008.

[15] S. L. Handley, T. L. Dunn, G. Waldron, and J. M. Baker, "Tryptophan, cortisol and puerperal mood," British Journal of Psychiatry, vol. 136, no. 5, pp. 498-508, 1980.

[16] A. Feksi, B. Harris, R. F. Walker, D. Riad-Fahmy, and R. G. Newcombe, "Maternity blues' and hormone levels in saliva," Journal of Affective Disorders, vol. 6, no. 3-4, pp. 351-355, 1984.

[17] V. Kuevi, R. Causon, A. F. Dixson et al., "Plasma amine and hormone changes in 'post-partum blues,' Clinical Endocrinology, vol. 19, no. 1, pp. 39-46, 1983.

[18] M.-A. Magiakou, G. Mastorakos, D. Rabin, B. Dubbert, P. W. Gold, and G. P. Chrousos, "Hypothalamic corticotropinreleasing hormone suppression during the postpartum period: implications for the increase in psychiatric manifestations at this time," Journal of Clinical Endocrinology and Metabolism, vol. 81, no. 5, pp. 1912-1917, 1996.

[19] I. S. Yim, L. M. Glynn, C. D. Schetter, C. J. Hobel, A. ChiczDeMet, and C. A. Sandman, "Risk of postpartum depressive symptoms with elevated corticotropin-releasing hormone in human pregnancy," Archives of General Psychiatry, vol. 66, no. 2, pp. 162-169, 2009.

[20] P. A. Brennan, R. Pargas, E. F. Walker, P. Green, D. J. Newport, and Z. Stowe, "Maternal depression and infant cortisol: influences of timing, comorbidity and treatment," Journal of Child Psychology and Psychiatry, vol. 49, no. 10, pp. 1099-1107, 2008.

[21] K. Priebe, R. D. Romeo, D. D. Francis et al., "Maternal influences on adult stress and anxiety-like behavior in C57BL/6J and BALB/cJ mice: a cross-fostering study," Developmental Psychobiology, vol. 47, pp. 398-407, 2005.

[22] S. Brummelte, S. E. Lieblich, and L. A. M. Galea, "Gestational and postpartum corticosterone exposure to the dam affects behavioral and endocrine outcome of the offspring in a sexually-dimorphic manner," Neuropharmacology, vol. 62, no. 1, pp. 406-418, 2012.

[23] W. E. Cullinan, D. R. Ziegler, and J. P. Herman, "Functional role of local GABAergic influences on the HPA axis," Brain Structure and Function, vol. 213, no. 1-2, pp. 63-72, 2008.

[24] J. P. Herman and W. E. Cullinan, "Neurocircuitry of stress: central control of the hypothalamo-pituitary-adrenocortical axis," Trends in Neurosciences, vol. 20, no. 2, pp. 78-84, 1997.

[25] J. P. Herman, N. K. Mueller, and H. Figueiredo, "Role of GABA and glutamate circuitry in hypothalamo-pituitaryadrenocortical stress integration," Annals of the New York Academy of Sciences, vol. 1018, pp. 35-45, 2004.

[26] J. P. Herman, J. G. Tasker, D. R. Ziegler, and W. E. Cullinan, "Local circuit regulation of paraventricular nucleus stress integration: glutamate-GABA connections," Pharmacology Biochemistry and Behavior, vol. 71, no. 3, pp. 457-468, 2002.

[27] J. Sarkar, S. Wakefield, G. MacKenzie, S. J. Moss, and J. Maguire, "Neurosteroidogenesis is required for the physiological response to stress: role of neurosteroid-sensitive GABA A receptors," The Journal of Neuroscience, vol. 31, no. 50, pp. 1819818210, 2011.

[28] B. G. Gunn, L. Cunningham, M. A. Cooper et al., "Dysfunctional astrocytic and synaptic regulation of hypothalamic glutamatergic transmission in a mouse model of early-life adversity: relevance to neurosteroids and programming of the stress response," Journal of Neuroscience, vol. 33, no. 50, pp. 19534-19554, 2013.

[29] D. Misdrahi, M. C. Pardon, F. Pérez-Diaz, N. Hanoun, and C. Cohen-Salmon, "Prepartum chronic ultramild stress increases corticosterone and estradiol levels in gestating mice: implications for postpartum depressive disorders," Psychiatry Research, vol. 137, no. 1-2, pp. 123-130, 2005.

[30] M.-C. Pardon, P. Gérardin, C. Joubert, F. Pérez-Diaz, and C. Cohen-Salmon, "Influence of prepartum chronic ultramild stress on maternal pup care behavior in mice," Biological Psychiatry, vol. 47, no. 10, pp. 858-863, 2000.

[31] R. D. Porsolt, A. Bertin, and M. Jalfre, "Behavioral despair in mice: a primary screening test for antidepressants," Archives Internationales de Pharmacodynamie et de Therapie, vol. 229, no. 2, pp. 327-336, 1977.

[32] M. L. Barbaccia, G. Roscetti, M. Trabucchi et al., "Timedependent changes in rat brain neuroactive steroid concentrations and GABA(A) receptor function after acute stress," Neuroendocrinology, vol. 63, no. 2, pp. 166-172, 1996.

[33] J. Maguire and I. Mody, "Neurosteroid synthesis-mediated regulation of GABAA receptors: relevance to the ovarian cycle and stress," The Journal of Neuroscience, vol. 27, no. 9, pp. 21552162, 2007.

[34] V. Lee, J. Sarkar, and J. Maguire, "Loss of Gabrd in CRH neurons blunts the corticosterone response to stress and diminishes stress-related behaviors," Psychoneuroendocrinology, vol. 41, pp. 75-88, 2014.

[35] S. Miller and J. Maguire, "Deficits in KCC2 and activation of the HPA axis lead to depressionlike behavior following social defeat," Hormonal Studies, vol. 2, no. 1, 2014.

[36] K. K. O’Toole, A. Hooper, S. Wakefield, and J. Maguire, "Seizure-induced disinhibition of the HPA axis increases seizure susceptibility," Epilepsy Research, vol. 108, no. 1, pp. 2943, 2014.

[37] E. J. Nestler, E. Gould, H. Manji et al., "Preclinical models: status of basic research in depression," Biological Psychiatry, vol. 52, pp. 503-528, 2002.

[38] J. W. Rich-Edwards, K. Kleinman, A. Abrams et al., "Sociodemographic predictors of antenatal and postpartum depressive symptoms among women in a medical group practice," Journal of Epidemiology and Community Health, vol. 60, no. 3, pp. 221227, 2006.

[39] C. B. Ballinger, M. C. K. Browning, and A. H. W. Smith, "Hormone profiles and psychological symptoms in peri-menopausal women," Maturitas, vol. 9, no. 3, pp. 235-251, 1987.

[40] A. Heidrich, M. Schleyer, H. Spingler et al., "Postpartum blues: relationship between not-protein bound steroid hormones in plasma and postpartum mood changes," Journal of Affective Disorders, vol. 30, no. 2, pp. 93-98, 1994.

[41] M. W. O’Hara, J. A. Schlechte, D. A. Lewis, and M. W. Varner, "Controlled prospective study of postpartum mood disorders: psychological, environmental, and hormonal variables," Journal of Abnormal Psychology, vol. 100, no. 1, pp. 63-73, 1991.

[42] V. Hendrick, L. L. Altshuler, and R. Suri, "Hormonal changes in the postpartum and implications for postpartum depression," Psychosomatics, vol. 39, no. 2, pp. 93-101, 1998.

[43] B. Harris, S. Johns, H. Fung et al., "The hormonal environment of post-natal depression," British Journal of Psychiatry, vol. 154, pp. 660-667, 1989.

[44] G. Stein, J. Morton, A. Marsh, J. Hartshorn, J. Ebeling, and U. Desaga, "Vasopressin and mood during the puerperium," Biological Psychiatry, vol. 19, no. 12, pp. 1711-1717, 1984.

[45] G. P. Chrousos, D. J. Torpy, and P. W. Gold, "Interactions between the hypothalamic-pituitary-adrenal axis and the female reproductive system: clinical implications," Annals of Internal Medicine, vol. 129, no. 3, pp. 229-240, 1998. 
[46] B. J. Carroll, F. Cassidy, D. Naftolowitz et al., "Pathophysiology of hypercortisolism in depression," Acta Psychiatrica Scandinavica, vol. 115, no. 433, pp. 90-103, 2007.

[47] A. J. Douglas, S. L. Meddle, N. Toschi, O. J. Bosch, and I. D. Neumann, "Reduced activity of the noradrenergic system in the paraventricular nucleus at the end of pregnancy: implications for stress hyporesponsiveness," Journal of Neuroendocrinology, vol. 17, no. 1, pp. 40-48, 2005.

[48] Z. N. Stowe and C. B. Nemeroff, "Women at risk for postpartumonset major depression," American Journal of Obstetrics and Gynecology, vol. 173, no. 2, pp. 639-645, 1995.

[49] L. K. Takahashi, J. G. Turner, and N. H. Kalin, "Prolonged stressinduced elevation in plasma corticosterone during pregnancy in the rat: implications for prenatal stress studies," Psychoneuroendocrinology, vol. 23, no. 6, pp. 571-581, 1998.

[50] S. Brummelte and L. A. M. Galea, "Chronic corticosterone during pregnancy and postpartum affects maternal care, cell proliferation and depressive-like behavior in the dam," Hormones and Behavior, vol. 58, no. 5, pp. 769-779, 2010.

[51] H. Zahwa, J. L. Yorty, and R. H. Bonneau, "Elevated maternal corticosterone during lactation hinders the neonatal adaptive immune response to herpes simplex virus (HSV) infection," Brain, Behavior, and Immunity, vol. 22, no. 3, pp. 339-353, 2008.

[52] L. Murray and P. J. Cooper, "Effects of postnatal depression on infant development," Archives of Disease in Childhood, vol. 77, no. 2, pp. 99-101, 1997.

[53] C. de Weerth, Y. van Hees, and J. K. Buitelaar, "Prenatal maternal cortisol levels and infant behavior during the first 5 months," Early Human Development, vol. 74, no. 2, pp. 139-151, 2003.

[54] S. L. Halligan, J. Herbert, I. M. Goodyer, and L. Murray, "Exposure to postnatal depression predicts elevated cortisol in adolescent offspring," Biological Psychiatry, vol. 55, no. 4, pp. 376-381, 2004.

[55] S. Brummelte, K. L. Schmidt, M. D. Taves, K. K. Soma, and L. A. M. Galea, "Elevated corticosterone levels in stomach milk, serum, and brain of male and female offspring after maternal corticosterone treatment in the rat," Developmental Neurobiology, vol. 70, no. 10, pp. 714-725, 2010.

[56] T. Pearlstein, M. Howard, A. Salisbury, and C. Zlotnick, "Postpartum depression," American Journal of Obstetrics and Gynecology, vol. 200, no. 4, pp. 357-364, 2009.

[57] R. Priestnall, "Effects of litter size on the behaviour of lactating female mice (Mus musculus)," Animal Behaviour, vol. 20, no. 2, pp. 386-394, 1972.

[58] P. F. D. Seitz, “The effects of infantile experiences upon adult behavior in animal subjects: I. Effects of litter size during infancy upon adult behavior in the rat," The American Journal of Psychiatry, vol. 110, no. 12, pp. 916-927, 1954. 

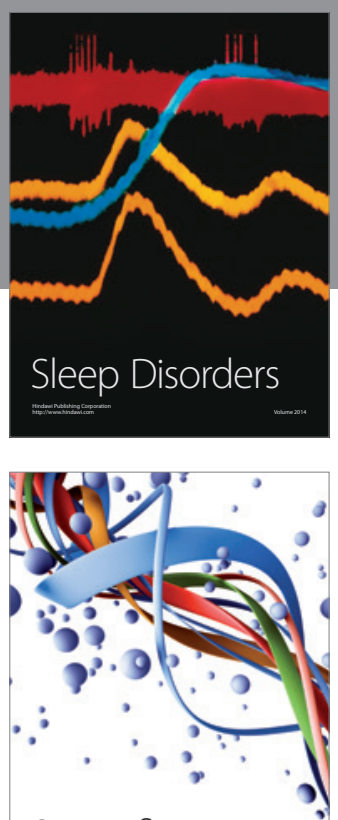

Scientifica
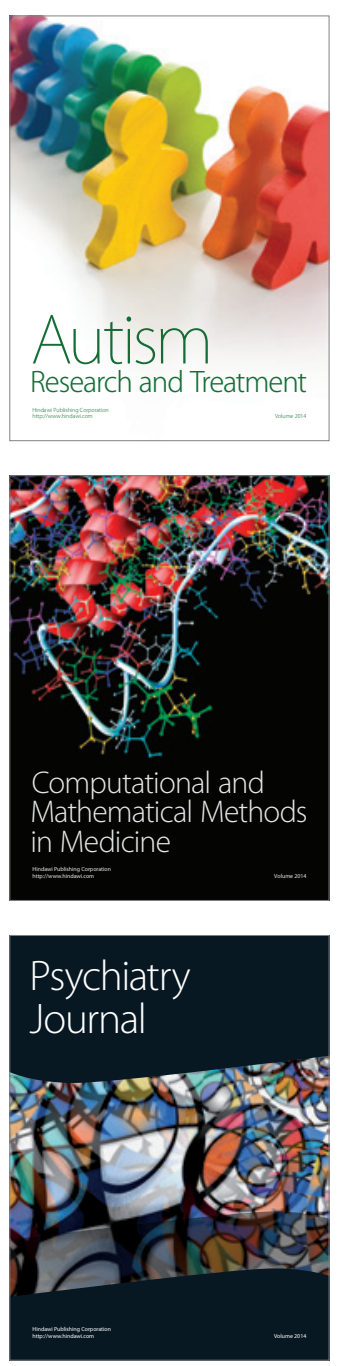
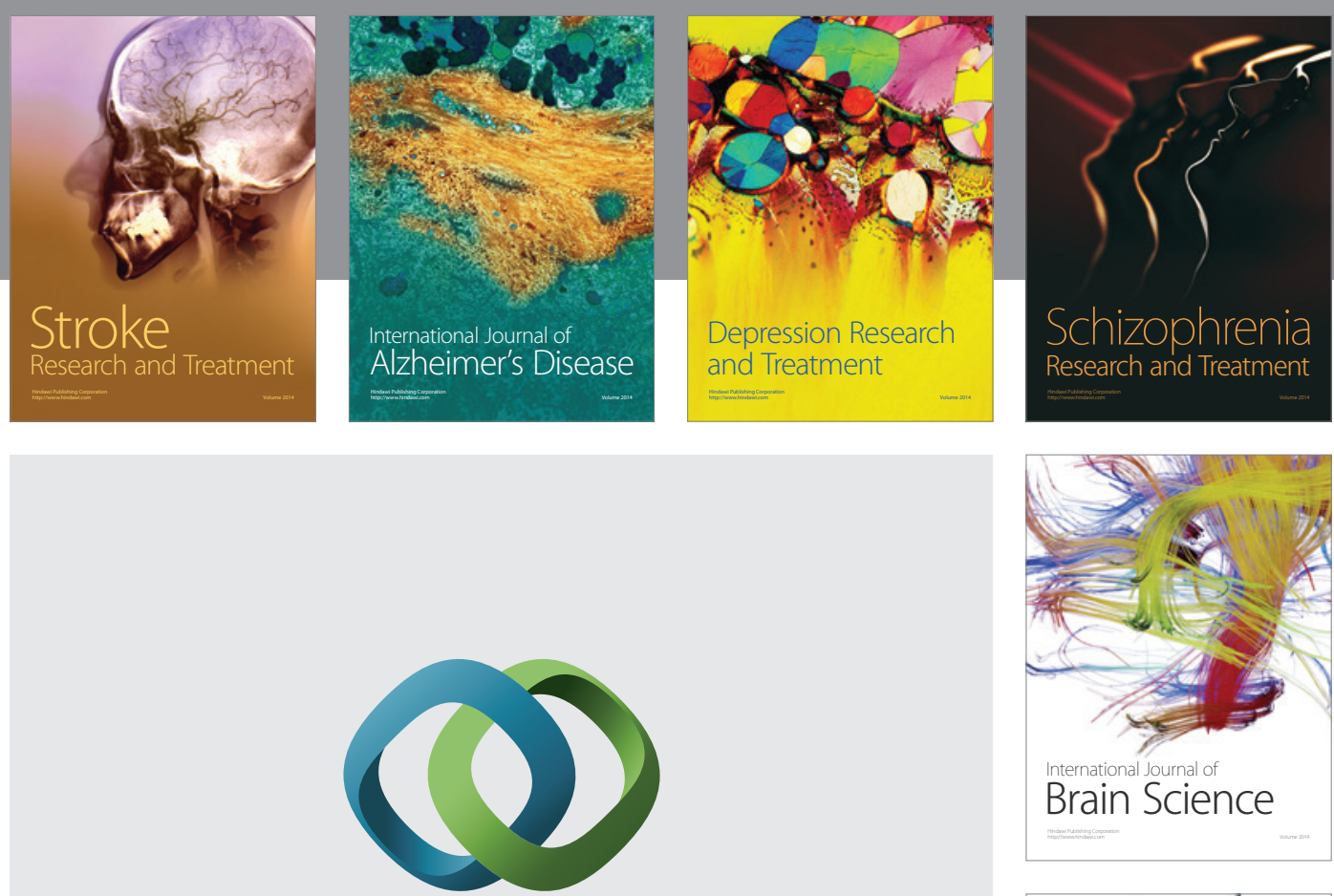

\section{Hindawi}

Submit your manuscripts at

http://www.hindawi.com
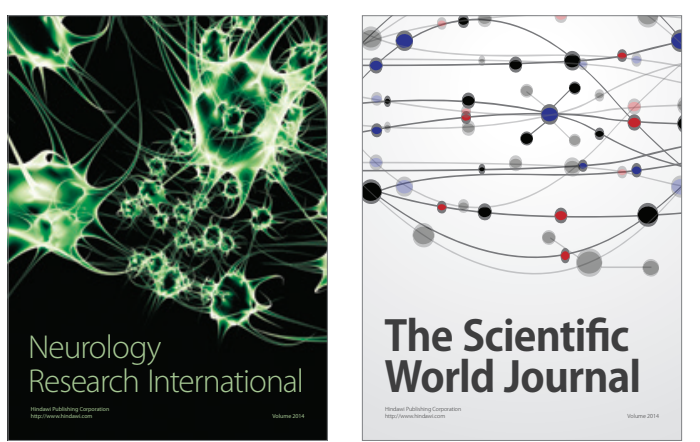

The Scientific World Journal

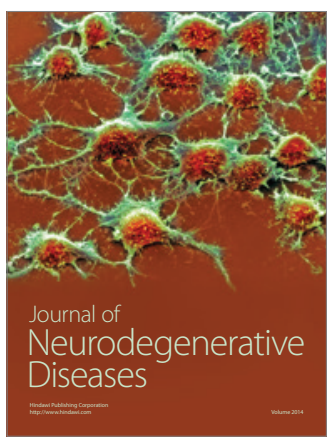

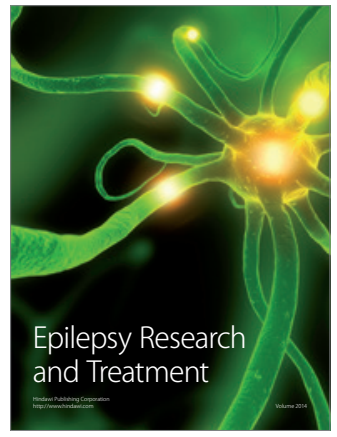

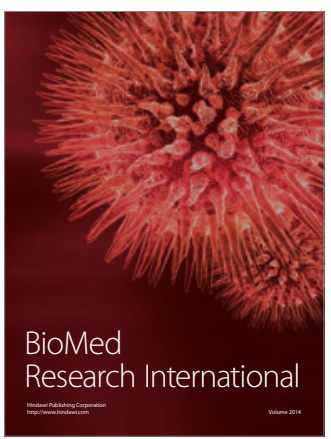

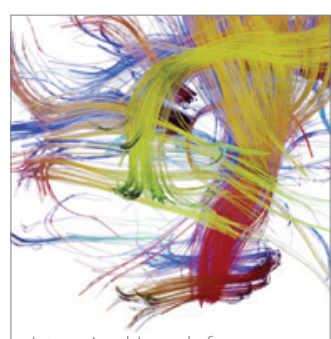

Brain Science

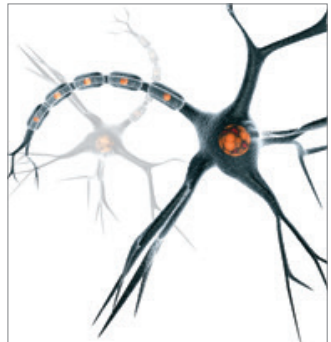

Neural Plasticity
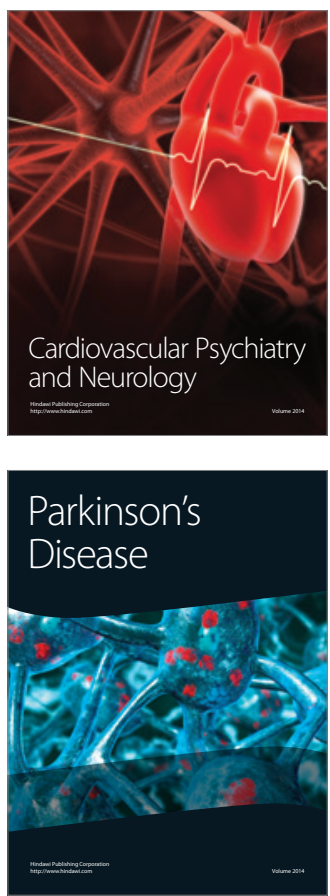